\title{
Global Criterion Method Based on Principal Components to the Optimization of Manufacturing Processes with Multiple Responses
}

\author{
José Henrique de Freitas Gomes - Aluizio Ramos Salgado Júnior - Anderson Paulo de Paiva - \\ João Roberto Ferreira - Sebastião Carlos da Costa - Pedro Paulo Balestrassi* \\ Federal University at Itajuba, Institute of Production Engineering and Management, Brazil
}

\begin{abstract}
The necessity of efficient and controlled processes has increased the demand by employing optimization methods to the most diverse industrial processes. For these cases, the Global Criterion Method is described in literature as a technique indicated for multi-objective optimizations. However, if the problem presents correlations between the responses, this technique does not consider such information. In this context, the Principal Component Analysis is a multivariate tool that can be used to represent correlated responses by uncorrelated components. Given that to negligence the correlation structure between the responses increases the likelihood of the optimization method in finding an inappropriate optimum point, the objective of this work is to combine the GCM and PCA in a strategy able to deal with problems having multiple correlated responses. For this reason, such strategy was used to optimize the $12 L 14$ free machining steel turning process, characterized as an important machining operation. The optimized responses included the mean roughness, total roughness, cutting time and material removal rate. As input parameters, the cutting speed, feed rate and depth of cut were considered. Response Surface Methodology was employed to build the objective functions. The GCM based on principal components was successfully applied, presenting better practical results and a more appropriate location of the optimal point in comparison to the conventional GCM.
\end{abstract}

Keywords: multi-objective optimization, global criterion method, principal component analysis, free machining steel turning

\section{O INTRODUCTION}

In industrial environments, it is becoming more and more important that items can be produced to satisfy several requirements simultaneously, and many of them are related to its cost, quality and productivity. Thus, considering that the manufacturing processes must be configured to obtain the best results for a set of characteristics, the interest in employing multiobjective optimization techniques has been increasing [1] and [2].

Among the optimization methods contemplating multiple responses, the desirability function [3], the multivariate integration [4] and the capacity indexes MCpm and MCpk [5] and [6] are listed as examples. Rao [7] describes the Global Criterion Method (GCM) as an interesting strategy. According to the author, the multiple objectives are optimized at the same time when the individual objective functions are combined in only one function, defined as the global optimization criterion of the process.

However, if the problem presents multiple correlated characteristics, the Global Criterion Method, as well as other conventional techniques, does not consider the correlation structure between the responses. This negligence, according to some researchers, may conduct the results to inadequate optimum points [8] and [9]. Paiva et al. [10] argue that the transfer functions used to represent the process outputs are strongly influenced by significant correlations existing between the responses of interest. Therefore, considering that the mathematical models are of great importance to the problem formulation, non-consideration of the correlation structure will affect the optimal point location.

In attempt to offer a more adequate treatment to the optimization problems with multiple correlated responses, the Principal Component Analysis (PCA) has been shown as a good alternative [11] and [12]. The PCA consists in a multivariate statistical tool that concerns in explaining the variance-covariance structure of a data set, using linear combinations of the original variables. Thus, the original correlated responses are represented by new uncorrelated variables, called principal components.

Given that the Global Criterion Method is presented as a technique to multi-objective optimizations but does not take into account the correlation structure between the responses, the objective of this work is to incorporate the PCA in the original formulation for GCM described by Rao [7], and verify how this analysis influences in determining the optimal solution. For this, such techniques were applied on the $12 \mathrm{~L} 14$ free machining steel turning process, characterized as one important operation in the modern industry.

Nevertheless, the study of manufacturing processes by optimization tools require that the mathematical relationships between the input parameters and the process responses be known. Therefore, before the optimization itself, such functions were modeled through Response Surface Methodology. 


\section{THEORETICAL FRAMEWORK}

\subsection{Response Surface Methodology (RSM)}

According to Montgomery [13], RSM is a collection of mathematical and statistical techniques that are useful for the modeling and analysis of problems in which a response of interest is influenced by several variables and the objective is to optimize this response.

The second order polynomial developed for a response surface that relates a given response $y$ with $k$ input variables presents the following format described by Eq. (1):

$$
y=\beta_{0}+\sum_{i=1}^{k} \beta_{i} x_{i}+\sum_{i=1}^{k} \beta_{i i} x_{i}^{2}+\sum \sum_{i<j} \beta_{i j} x_{i} x_{j},
$$

where $y$ is the response of interest, $x_{i}$ are the input parameters, $\beta_{0}, \beta_{i}, \beta_{i i}, \beta_{i j}$ are the coefficients to be estimated, and $k$ is the number of input parameters considered.

To estimate the coefficients stated in Eq. (1), the Ordinary Least Squares is the typically used algorithm. After the model building, the ANOVA statistical procedure is usually employed to check its significance and its adjustment.

\subsection{Global Criterion Method (GCM)}

A multi-objective optimization problem is one that, considering inequality constraints, can be stated as Eq. (2):

$$
\begin{array}{cl}
\text { Minimize } & f_{1}(\mathbf{x}), f_{2}(\mathbf{x}), \ldots, f_{p}(\mathbf{x}) \\
\text { Subject to }: & g_{j}(\mathbf{x}) \leq 0, j=1,2, \ldots, m
\end{array}
$$

where $f_{i}(\mathbf{x})$ are objective functions, and $g_{j}(\mathbf{x})$ constraints.

However, under various circumstances, the multiple responses considered in a process present conflict of objectives, with individual optimization leading to different solution sets. For this kind of problem, Rao [7] characterizes the Global Criterion Method as a strategy where the optimal solution is found by minimizing a preselected global criterion, $F(\mathbf{x})$, such as the sum of the squares of the relative deviations of the individual objective functions from the feasible ideal solutions. The GCM formulation is given by:

$$
\text { Minimize } \quad F(\mathbf{x})=\sum_{i=1}^{p}\left\{\frac{T_{i}-f_{i}(\mathbf{x})}{T_{i}}\right\}^{2},
$$

where $F(\mathbf{x})$ is the global criterion, $T_{i}$ is the target defined for the $i^{\text {th }}$ objective, $f_{i}(\mathbf{x})$ are objective functions, $g_{j}(\mathbf{x})$ are constraints, and $p$ is number of objectives.

Thus, with targets defined for each response of interest, the multiple objectives are combined into an only function, which becomes the global optimization function for the process.

To obtain the optimal point from GCM formulation, several optimization algorithms can be applied. In this work, the Genetic Algorithm was used because it is considered an effective algorithm to global optimizations [14] and [15].

\subsection{Principal Component Analysis (PCA)}

Suppose that the objective functions $f_{1}(\mathbf{x}), f_{2}(\mathbf{x}), \ldots$, $f_{p}(\mathbf{x})$, presented in Eqs. (2) and (3), are correlated with values written in terms of a random vector $\mathbf{Y}^{T}=\left[Y_{1}, Y_{2}, \ldots, Y_{p}\right]$. Assuming that $\Sigma$ is the variancecovariance matrix associated to this vector, then $\Sigma$ can be factorized in pairs of eigenvalues-eigenvectors $\left(\lambda_{i}, e_{i}\right), \ldots, \geq\left(\lambda_{p}, e_{p}\right)$, where $\lambda_{1} \geq \lambda_{1} \geq \ldots \geq \lambda_{p} \geq 0$, such as the $i^{\text {th }}$ uncorrelated linear combination may be stated as $P C_{i}=e_{i}^{T} \mathbf{Y}=e_{1 i} Y_{1}+e_{2 i} Y_{2}+\ldots+e_{p i} Y_{p}$, with $i=1,2, \ldots, p$. The $i^{\text {th }}$ principal component can be obtained as maximization of this linear combination [16]. This statistical technique is called Principal Component Analysis (PCA), one of the most widely applied tools to summarize common patterns of variation among variables retaining meaningful information in the early PCA axes [17] and [18]. The geometric interpretation of these axes is shown in Fig. 1.

Generally, as the parameters $\sum e \rho$ are unknown, the sample correlation matrix $R_{i j}$ and the sample variance-covariance matrix $S_{i j}$ may be used [16]. If the variables studied are taken in the same system of units or if they are previously standardized, $S_{i j}$ is a more appropriate choice. Otherwise, $R_{i j}$ must be employed in the factorization. The sample variance-covariance matrix can be written as follows:

$$
\begin{gathered}
S_{i j}=\left[\begin{array}{cccc}
s_{11} & s_{12} & \cdots & s_{1 p} \\
s_{21} & s_{22} & \cdots & s_{21} \\
\vdots & \vdots & \ddots & \vdots \\
s_{p 1} & s_{p 2} & \cdots & s_{p p}
\end{array}\right], \\
s_{i i}=\frac{1}{n} \sum_{j=1}^{n}\left(y_{i}-\bar{y}_{i}\right)^{2}, s_{i j}=\frac{1}{n} \sum_{j=1}^{n}\left(y_{i}-\bar{y}_{i}\right)\left(y_{j}-\bar{y}_{j}\right) .
\end{gathered}
$$


Then, the elements of sample correlation matrix $R_{i j}$ can be obtained as:

$$
\begin{aligned}
r_{\left(y_{i}, y_{j}\right)} & =\frac{\operatorname{Cov}\left(y_{i}, y_{j}\right)}{\sqrt{\operatorname{Var}\left(y_{i}\right) \times \operatorname{Var}\left(y_{j}\right)}}=\frac{\hat{e}_{i j} \sqrt{\hat{\lambda}_{i}}}{\sqrt{s_{i i}}}= \\
& =\frac{s_{i j}}{\sqrt{s_{i i} \times s_{j j}}}, \quad i, j=1,2, \ldots, p .
\end{aligned}
$$

In practical terms, the principal component $(P C)$ is an uncorrelated linear combination expressed in terms of a score matrix, defined as:

$$
P C_{k}=\mathbf{Z}^{\mathbf{T}} \mathbf{E}=\left[\begin{array}{cccc}
\left(\frac{x_{11}-\bar{x}_{1}}{\sqrt{s_{11}}}\right) & \left(\frac{x_{21}-\bar{x}_{2}}{\sqrt{s_{22}}}\right) & \cdots & \left(\frac{x_{p 1}-\bar{x}_{p}}{\sqrt{s_{p p}}}\right) \\
\left(\frac{x_{12}-\bar{x}_{1}}{\sqrt{s_{11}}}\right) & \left(\frac{x_{22}-\bar{x}_{2}}{\sqrt{s_{22}}}\right) & \cdots & \left(\frac{x_{p 2}-\bar{x}_{p}}{\sqrt{s_{p p}}}\right) \\
\vdots & \ddots & \vdots \\
\vdots & \vdots
\end{array}\right]^{\mathbf{T}} \times\left[\begin{array}{cccc}
e_{11} & e_{12} & \cdots & e_{1 p} \\
e_{21} & e_{22} & \cdots & e_{2 p} \\
\vdots & \vdots & \ddots & \vdots \\
e_{1 p} & e_{2 p} & \cdots & e_{p p}
\end{array}\right] .
$$

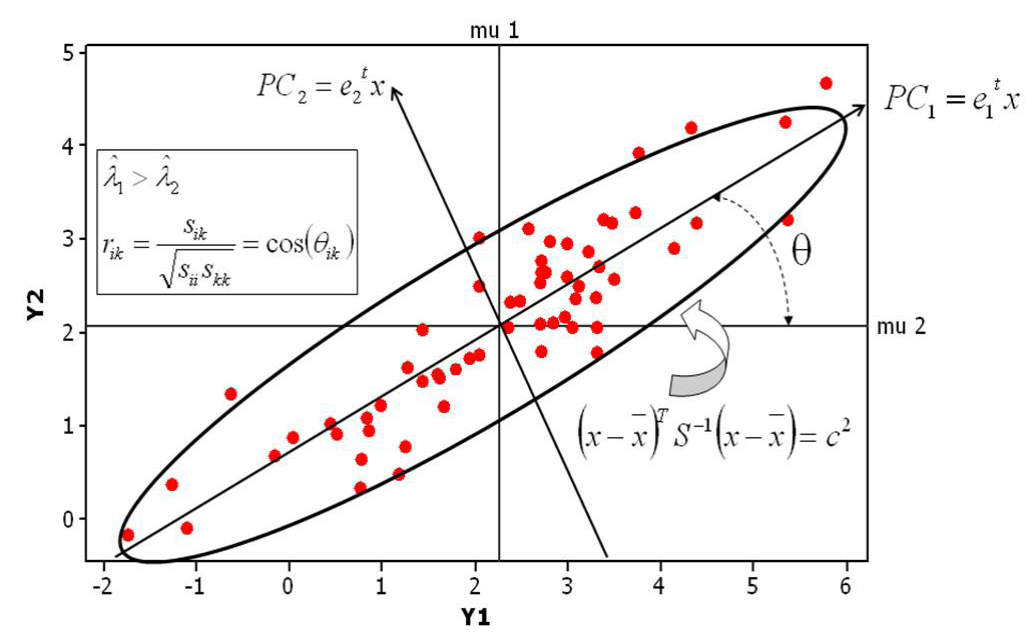

Fig. 1. Geometric interpretation of principal components

\subsection{Global Criterion Method Based on Principal Components}

The global criterion $F(\mathbf{x})$, as stated by Eq. (3), is formulated from the objective functions and the targets defined to each response of interest. If the objective functions are unknown, then they can be modeled by RSM from experimental data. However, when the responses are correlated, this strategy does not take into account the correlation structure between them.
On the other hand, it has been seen in previous section that the principal components are characterized as uncorrelated representations of original correlated variables.

Considering that the principal components, through their scores, can also be modeled by RSM as functions of input parameters [10], then the Global Criterion Method based on principal components is written as: 
Minimize $\quad F_{P C}(\mathbf{x})=\sum_{i=1}^{k}\left\{\frac{\zeta_{P C i}-P C_{i}(\mathbf{x})}{\zeta_{P C i}}\right\}^{2}$,

Subject to : $\quad g_{j}(\mathbf{x}) \leq 0, \quad j=1,2, \ldots, m$

where $F_{P C}(\mathbf{x})$ is the global criterion based on principal components, $\zeta_{P C i}$ is the target defined for the $i^{\text {th }}$ principal component, $P C_{i}(\mathbf{x})$ are quadratic models developed for the principal components, $g_{j}(\mathbf{x})$ are constraints, and $k$ is the number of principal components considered.

The determination of the targets for principal components requires that the targets for original responses are previously defined. The $\zeta_{P C i}$ is then calculated as a linear combination between the eigenvectors of principal components and the standardized original responses in relation to their targets. This procedure is showed by Eqs. (8) and (9).

$$
\zeta_{P C i}=\sum_{j=1}^{p} e_{j i} \cdot Z\left(y_{j} \mid \zeta_{y_{j}}\right),
$$

where $\zeta_{P C i}$ is the target defined for the $i^{\text {th }}$ principal component, $p$ is the number of objectives, $e_{j i}$ are coefficients of the principal components' eigenvectors, and $Z\left(y_{j} \mid \zeta_{y j}\right)$ are the standardized original responses in relation to their targets, calculated as:

$$
Z\left(y_{j} \mid \zeta_{y_{j}}\right)=\frac{\zeta_{y_{j}}-\bar{y}_{j}}{\sigma_{y_{j}}}
$$

where $\zeta_{y_{j}}$ are targets defined for the original responses, $\bar{y}_{j}$ are the means of responses, and $\sigma_{y_{j}}$ are the standard deviations of responses.

Analogously to the Eq. (3), the obtaining of optimal point for the formulation given in Eq. (7) is done by employing optimization algorithms. The Genetic Algorithm was also used in this work for this purpose.

Finally, for the Global Criterion Method based on principal components, it is important to highlight that this strategy combines the main advantages offered by GCM and ACP, since it continues being a technique for multi-objective optimizations, but now considering the correlation structure existing between the responses.

\section{OPTIMIZATION OF THE 12L14 FREE MACHINING STEEL TURNING}

With the aim of verifying the functionality of the GCM based on principal components in improving performance of manufacturing processes, such strategy was applied to the optimization of 12L14 free machining steel turning process.

This is described as a relevant operation within the current industrial context, since the free machining steels are developed to offer good machining conditions and excellent chip formations. For this process, other mechanical characteristics, as ductility, strength and response to heat treatments are considered as secondary factors. The free machining steels have been employed in production of elements that do not need to present structural responsibility, as appliances and components to pumps, plugs and connections.

Due to the fact that mechanical properties are not the most important requirements for the 12L14 free machining steel turning process, its optimization is mainly concerned with its productivity and surface quality. The surface quality was then optimized through the mean roughness $(R a)$ and total roughness $(R t)$. For the productivity, cutting time $(C t)$ and material removal rate $(M R R)$ were the optimized characteristics. The cutting speed $(V)$, feed rate $(f)$ and depth of cut $(d)$ were considered as input parameters.

Given that the objective functions between the input parameters and responses were initially unknown, such relationships were modeled using RSM. Thus, data were collected from turning experiments performed with work pieces of $12 \mathrm{~L} 14$ free machining steel $(0.09 \% \mathrm{C} ; 0.03 \% \mathrm{Si} ; 1.24 \% \mathrm{Mn}$; $0.046 \% \mathrm{P} ; 0.273 \% \mathrm{~S} ; 0.15 \% \mathrm{Cr} ; 0.08 \% \mathrm{Ni} ; 0.26 \%$ $\mathrm{Cu} ; 0.001 \% \mathrm{Al} ; 0.02 \% \mathrm{Mo} ; 0.28 \% \mathrm{~Pb} ; 0.0079 \%$ N2), with dimensions of $\phi 40 \times 295 \mathrm{~mm}$. The machine tool used was a NARDINI CNC lathe, with $7.5 \mathrm{cv}$ power and maximum rotation of 4,000 rpm. The hard metal inserts (ISO P35 code SNMG 090304 - PM, Sandvik class GC 4035) were coated with three layers (Ti(C.N), $\mathrm{Al}_{2} \mathrm{O}_{3}, \mathrm{TiN}$ ) and a tool holder ISO code DSBNL 1616H09 was employed.

A central composite design with three factors at two levels $\left(2^{k}=2^{3}=8\right)$, six axial points $(2 k=6)$ and three center points was chosen as experimental matrix, which resulted in 17 experiments. The adopted value for axial distance $\alpha$ was 1.682 . Table 1 presents the range defined for input parameters. To record the responses, mean roughness and total roughness were measured by a roughmeter. Cutting time and material removal rate were calculated. At the end of experiments, the experimental matrix (Table 2) was built. 
Table 1. Parameters and their levels

\begin{tabular}{|c|c|c|c|c|c|c|c|}
\hline \multirow{2}{*}{ Parameter } & \multirow{2}{*}{ Symbol } & \multirow{2}{*}{ Unit } & \multicolumn{5}{|c|}{ Levels } \\
\hline & & & -1.682 & -1 & 0 & +1 & +1.682 \\
\hline Cutting speed & $V$ & {$[\mathrm{~m} / \mathrm{min}]$} & 180 & 220 & 280 & 340 & 380 \\
\hline Feed rate & $f$ & [mm/rev] & 0.07 & 0.08 & 0.10 & 0.12 & 0.13 \\
\hline Depth of cut & $d$ & {$[\mathrm{~mm}]$} & 0.53 & 0.70 & 0.95 & 1.20 & 1.37 \\
\hline
\end{tabular}

Table 2. Experimental matrix

\begin{tabular}{cccccccc}
\hline \multirow{2}{*}{ Test } & \multicolumn{3}{c}{ Parameters } & \multicolumn{3}{c}{ Responses } \\
\cline { 2 - 7 } & $V[\mathrm{~m} / \mathrm{min}]$ & $F[\mathrm{~mm} / \mathrm{rev}]$ & $d[\mathrm{~mm}]$ & $R a[\mu \mathrm{m}]$ & $R t[\mu \mathrm{m}]$ & $C t[\mathrm{~min}]$ & $M R R[\mathrm{~cm} / \mathrm{min}]$ \\
\hline 1 & 220 & 0.08 & 0.70 & 1.36 & 9.49 & 2.11 & 12.32 \\
\hline 2 & 340 & 0.08 & 0.70 & 1.65 & 10.70 & 1.36 & 19.04 \\
\hline 3 & 220 & 0.12 & 0.70 & 1.78 & 10.08 & 1.40 & 18.48 \\
\hline 4 & 340 & 0.12 & 0.70 & 1.84 & 10.41 & 0.91 & 28.56 \\
\hline 5 & 220 & 0.08 & 1.20 & 2.22 & 14.71 & 2.11 & 21.12 \\
\hline 6 & 340 & 0.08 & 1.20 & 2.20 & 13.47 & 1.36 & 32.64 \\
\hline 7 & 220 & 0.12 & 1.20 & 1.82 & 11.13 & 1.40 & 31.68 \\
\hline 8 & 340 & 0.12 & 1.20 & 2.24 & 13.20 & 0.91 & 48.96 \\
\hline 9 & 180 & 0.10 & 0.95 & 1.90 & 12.51 & 2.06 & 17.10 \\
\hline 10 & 380 & 0.10 & 0.95 & 2.08 & 12.49 & 0.98 & 36.10 \\
\hline 11 & 280 & 0.07 & 0.95 & 1.85 & 10.73 & 1.89 & 18.62 \\
\hline 12 & 280 & 0.13 & 0.95 & 1.85 & 10.78 & 1.02 & 34.58 \\
\hline 13 & 280 & 0.10 & 0.53 & 1.68 & 8.89 & 1.32 & 14.84 \\
\hline 14 & 280 & 0.10 & 1.37 & 2.30 & 13.37 & 1.32 & 38.36 \\
\hline 15 & 280 & 0.10 & 0.95 & 2.32 & 12.57 & 1.32 & 26.60 \\
\hline 16 & 280 & 0.10 & 0.95 & 2.23 & 12.84 & 1.32 & 26.60 \\
\hline 17 & 280 & 0.10 & 0.95 & 2.26 & 12.92 & 1.32 & 26.60 \\
\hline
\end{tabular}

\section{RESULTS AND DISCUSSION}

\subsection{Modeling of Objective Functions}

Writing the response surface function stated in Eq. (1) for three parameters, the following expression is obtained:

$$
\begin{aligned}
y= & \beta_{0}+\beta_{1} V+\beta_{2} f+\beta_{3} d+\beta_{11} V^{2}+\beta_{22} f^{2}+ \\
& +\beta_{33} d^{2}+\beta_{12} V f+\beta_{13} V d+\beta_{23} f d .
\end{aligned}
$$

To estimate the coefficients defined in Eq. (10), the statistical software Minitab ${ }^{\circledR}$ was employed and, from the experimental data presented in Table 2, the full quadratic models were developed for each response of interest. Then, the significance of models was tested through ANOVA procedure. Table 3 presents the coefficients for full quadratic models and the main results of ANOVA.

From Table 3 it can be observed that, considering a significance level of $95 \%$, all models are adequate, since $p$-values were lower than 0.05. Furthermore, the adj. $R^{2}$ values indicate high adjustments for the models, which means these expressions are reliable in representing the responses.

Finally, after non significant coefficients have been removed, the final models, or the objective functions for responses, were obtained. Eqs. (11) to (14) present these expressions.

$$
\begin{gathered}
R a=2.272+0.077 V+0.018 f+0.212 d- \\
-0.107 V^{2}-0.157 f^{2}-0.106 d^{2}-0.123 f d, \\
R t=12.708+0.172 V-0.254 f+1.418 d \\
-0.643 f^{2}-0.511 d^{2}+0.302 V f-0.518 f d, \\
C t=1.325-0.315 V-0.277 f+0.070 V^{2} \\
\quad+0.047 f^{2}+0.062 V f \quad, \\
M R R=26.600+5.679 V+5.082 f+6.997 d+ \\
\quad+1.140 V f+1.500 V d+1.400 f d .
\end{gathered}
$$


Table 3. Estimated coefficients for full quadratic models

\begin{tabular}{lcccc}
\hline \multirow{2}{*}{ Coeff. } & \multicolumn{4}{c}{ Responses } \\
\cline { 2 - 5 } & $\mathrm{Ra}$ & $\mathrm{Rt}$ & $\mathrm{Ct}$ & $\mathrm{MRR}$ \\
\hline$\beta_{0}$ & 2.272 & 12.757 & 1.324 & 26.600 \\
\hline$\beta_{1}$ & 0.077 & 0.172 & -0.315 & 5.679 \\
\hline$\beta_{2}$ & 0.018 & -0.254 & -0.277 & 5.082 \\
\hline$\beta_{3}$ & 0.212 & 1.418 & 0.000 & 6.997 \\
\hline$\beta_{11}$ & -0.107 & -0.038 & 0.070 & 0.000 \\
\hline$\beta_{22}$ & -0.157 & -0.655 & 0.048 & 0.000 \\
\hline$\beta_{33}$ & -0.106 & -0.522 & 0.002 & 0.000 \\
\hline$\beta_{12}$ & 0.026 & 0.301 & 0.062 & 1.140 \\
\hline$\beta_{13}$ & 0.006 & -0.090 & 0.000 & 1.500 \\
\hline$\beta_{23}$ & -0.123 & -0.518 & 0.000 & 1.400 \\
\hline$p$-value & 0.002 & 0.005 & 0.000 & 0.000 \\
\hline adj. $R^{2}[\%]$ & 85.46 & 80.63 & 99.68 & 99.72 \\
\hline
\end{tabular}

\subsection{Optimization by Conventional Global Criterion Method}

Before applying the GCM based on principal components to the optimization of $12 \mathrm{~L} 14$ free machining steel turning, this operation was also optimized by conventional GCM, with the aim of comparing both results.

By taking the objective functions developed for the process responses, the GCM formulation can be built. However, for this formulation, it is necessary that the targets of original responses are defined. These specifications were made by experts and took into account that the process application could be satisfied with good levels of surface quality and productivity. Table 4 show the targets defined for responses and their respective specification limits.

Thus, the optimization problem was built as stated in Eqs. (15) to (17). All characteristics were considered with the same degree of importance.

$$
\begin{aligned}
\operatorname{Min} G & =\left(\frac{1.5-R a}{1.5}\right)^{2}+\left(\frac{9.0-R t}{9.0}\right)^{2}+ \\
& +\left(\frac{1.2-C t}{1.2}\right)^{2}+\left(\frac{35-M R R}{35}\right)^{2},
\end{aligned}
$$

subject to: $\quad V, f, d \geq-1.682$,

$$
V, f, d \leq 1.682
$$

where $G$ is the global criterion, $R a, R t, C t, M R R$ are objective functions, and $V, f, d$ are the input parameters.

Finally, replacing $R a, R t, C t$ and $M R R$ in Eq. (15) by their respective objective functions, the final formulation of the problem was obtained given by:

$$
\begin{gathered}
\text { Min } G=\{[1.5-(2.272+0.077 V+0.018 f+ \\
+0.212 d-0.107 V^{2}-0.157 f^{2}-0.106 d^{2}- \\
-0.123 f d)] / 1.5\}^{2}+\{[9.0-(12.708+0.172 V \\
-0.254 f+1.418 d-0.643 f^{2}-0.511 d^{2}+ \\
+0.302 V f-0.518 f d)] / 9.0\}^{2}+\{[1.2- \\
-\left(1.325-0.315 V-0.277 f+0.070 V^{2}+\right. \\
\left.\left.\left.+0.047 f^{2}+0.062 V f\right)\right] / 1.2\right\}^{2}+\{[35- \\
-(26.600+5.679 V+5.082 f+6.997 d+ \\
+1.140 V f+1.500 V d+1.400 f d) / 35]\}^{2},
\end{gathered}
$$

subject to: $\quad V, f, d \geq-1.682$,

$$
V, f, d \leq 1.682 \text {. }
$$

Table 4. Targets and specification limits for responses

\begin{tabular}{lccc}
\hline Response & LSL & T & USL \\
\hline$R a$ & 1.0 & 1.5 & 2.0 \\
\hline$R t$ & 8.0 & 9.0 & 10.0 \\
\hline$C t$ & 1.0 & 1.2 & 1.4 \\
\hline$M R R$ & 30 & 35 & 40 \\
\hline
\end{tabular}

As can be observed, all optimized responses were established within the specification limits and relatively close to their targets, which suggests that it seems a good solution.

Table 5. Parameters used in Genetic Algorithm

\begin{tabular}{lc}
\hline Parameters & Values \\
\hline Iterations & 1,000 \\
\hline Convergence & 0.0001 \\
\hline Population size & 150 \\
\hline Mutation rate & 0.10 \\
\hline
\end{tabular}

Table 6. Optimal results for 12 L14 free machining steel turning obtained by conventional GCM

\begin{tabular}{lcccc}
\hline & \multicolumn{4}{c}{ Responses } \\
\cline { 2 - 5 } & $R a[\mu \mathrm{m}]$ & $R t[\mu \mathrm{m}]$ & $C t[\mathrm{~min}]$ & $M R R\left[\mathrm{~cm}^{3} / \mathrm{min}\right]$ \\
\hline Optimal & 1.53 & 9.72 & 1.28 & 36.4 \\
\hline Target & 1.50 & 9.00 & 1.20 & 35.0 \\
\hline
\end{tabular}

The optimal point was found by applying Genetic Algorithm in the previous formulation. Microsoft Excel $\AA$ was used for the mathematical programming of problem and the Solver Evolutionary supplement was employed. After some runs executed with random initial solutions and GA parameters given in Table 5, it was observed the optimal solution converged to the same point. Therefore, this was characterized as the global optimal point. Table 6 presents these results, 
obtained with a cutting speed of $218 \mathrm{~m} / \mathrm{min}$, feed rate of $0.13 \mathrm{~mm} / \mathrm{rev}$ and depth of cut of $1.24 \mathrm{~mm}$.

\subsection{Optimization by Global Criterion Method Based on Principal Components}

Table 7 presents the correlation structure for $R a$, $R t, C t$ and $M R R$. Since significant correlations were identified ( $p$-value less than 0.05), the application of GCM based on principal components as optimization strategy is justified.

Table 7. Correlation structure of the responses

\begin{tabular}{|c|c|c|c|}
\hline & Ra & $R t$ & $C t$ \\
\hline \multirow{2}{*}{$R t$} & 0.876 & & \\
\hline & 0.000 & & \\
\hline \multirow{2}{*}{$C t$} & -0.283 & 0.030 & \\
\hline & 0.272 & 0.909 & \\
\hline \multirow{2}{*}{ MRR } & 0.613 & 0.506 & -0.701 \\
\hline & 0.009 & 0.038 & 0.002 \\
\hline \multicolumn{4}{|c|}{ Cells: Pearson correlation } \\
\hline & & & \\
\hline
\end{tabular}

Table 8. Principal Component Analysis

\begin{tabular}{lcccc}
\hline & $P C 1$ & $P C 2$ & $P C 3$ & $P C 4$ \\
\hline Eigenvalues & 2.534 & 1.215 & 0.205 & 0.046 \\
\hline Proportion & 0.634 & 0.304 & 0.051 & 0.011 \\
\hline Cumulative & 0.634 & 0.937 & 0.989 & 1.000 \\
\hline Eigenvectors & $P C 1$ & $P C 2$ & $P C 3$ & $P C 4$ \\
\hline$R a$ & 0.570 & -0.289 & -0.552 & 0.536 \\
\hline$R t$ & 0.500 & -0.529 & 0.143 & -0.670 \\
\hline M & -0.347 & -0.732 & 0.421 & 0.409 \\
\hline
\end{tabular}

Performing the Principal Component Analysis for these responses (Table 8), it can be noticed that $93.7 \%$ of data are represented by two principal components. So, these new uncorrelated variables were used to substitute the original correlated responses.

Then, the objective functions for principal components were modeled taking the scores of each component obtained in the PCA. For this, the same procedure described in section 3.1 was employed. Eqs. (21) and (22) present such functions for $P C 1$ and $P C 2$, which showed adj. $R^{2}$ values of 94.18 and $92.81 \%$, respectively.

$$
\begin{aligned}
P C 1 & =0.991+0.808 V+0.491 f+1.265 d- \\
& -0.291 V^{2}-0.564 f^{2}-0.379 d^{2}+ \\
& +0.156 V f-0.331 f d,
\end{aligned}
$$

$$
\begin{aligned}
P C 2 & =-0.457+0.633 V+0.742 f- \\
& -0.452 d+0.289 f^{2}+0.279 d^{2}- \\
& -0.202 V f+0.342 f d .
\end{aligned}
$$

Through Eqs. (8) and (9), the PC's targets were calculated using data in Table 9. It resulted in values of $\mathbf{- 1 . 1 5 3}$ for $P C 1$ and $\mathbf{2 . 0 7 7}$ for $P C 2$.

Table 9. Used data to calculate the targets for principal components

\begin{tabular}{lcccc}
\hline & $R a$ & $R t$ & $C t$ & $M R R$ \\
\hline Mean & 1.974 & 11.781 & 1.419 & 26.600 \\
\hline Std. dev. & 0.280 & 1.622 & 0.396 & 9.707 \\
\hline Target & 1.5 & 9.0 & 1.2 & 35 \\
\hline Standardization & -1.692 & -1.715 & -0.554 & 0.865 \\
\hline Eigenvectors & $R a$ & $R t$ & $C t$ & $M R R$ \\
\hline$P C 1$ & 0.570 & 0.500 & -0.347 & 0.551 \\
\hline$P C 2$ & -0.289 & -0.529 & -0.732 & 0.318 \\
\hline
\end{tabular}

Finally, the formulation for GCM based on principal components was built, showing the following format:

Min $G_{P C}=\left(\frac{-1.153-P C 1}{-1.153}\right)^{2}+\left(\frac{2.077-P C 2}{2.077}\right)^{2}$,

subject to:

$$
\begin{gathered}
V, f, d \geq-1.682, \\
V, f, d \leq 1.682,
\end{gathered}
$$

where $G_{P C}$ is the global criterion based on principal components, $P C 1$ and $P C 2$ are objective functions for the principal components, and $V, f, d$ are the input parameters.

Replacing $P C 1$ and $P C 2$ in Eq. (23) by their objective functions, the final formulation is written as:

Min $G_{P C}=\{[-1.153-(0.991+0.808 V+$

$$
\begin{gathered}
+0.491 f+1.265 d-0.291 V^{2}-0.564 f^{2}- \\
\left.\left.-0.379 d^{2}+0.156 V f-0.331 f d\right)\right] / \\
/(-1.153)\}^{2}+\{[2.077-(-0.457+0.633 V+ \\
+0.742 f-0.452 d+0.289 f^{2}+0.279 d^{2}- \\
-0.202 V f+0.342 f d)] / 2.077\}^{2},
\end{gathered}
$$

subject to:

$$
\begin{gathered}
V, f, d \geq-1.682, \\
V, f, d \leq 1.682 .
\end{gathered}
$$

The Microsoft Excel ${ }^{\circledR}$ worksheet with Solver Evolutionary supplement and the Genetic Algorithm with parameters of Table 5 were also used to find the new global optimal point. These results are presented 
in Table 10, obtained for a cutting speed of $212 \mathrm{~m} /$ $\mathrm{min}$, feed rate of $0.13 \mathrm{~mm} / \mathrm{rev}$ and depth of cut of 1.33 $\mathrm{mm}$.

Table 10. Optimal results for $12 L 14$ free machining steel turning obtained by GCM based on principal components

\begin{tabular}{lcccc}
\hline & \multicolumn{4}{c}{ Responses } \\
\cline { 2 - 5 } & $R a[\mu \mathrm{m}]$ & Rt $[\mu \mathrm{m}]$ & Ct $[\mathrm{min}]$ & MRR $\left[\mathrm{cm}^{3} / \mathrm{min}\right]$ \\
\hline Optimal & 1.40 & 9.38 & 1.33 & 38.0 \\
\hline Target & 1.50 & 9.00 & 1.20 & 35.0 \\
\hline
\end{tabular}

Again, all optimized responses were established within the specification limits and relatively close to their targets. However, the global solution obtained with GCM based on principal components presented better surface finishing (lower roughness) and higher material removal. Although cutting time was higher, this solution was characterized as a more appropriate optimal point in relation to one obtained with the conventional GCM. Furthermore, this new optimal point was calculated taking into account the correlations between the original responses.

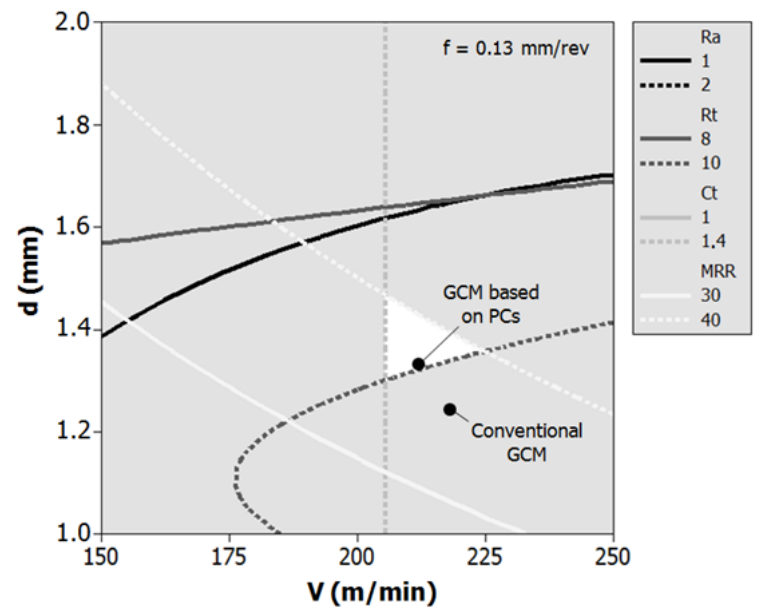

Fig. 2. Overlaid contour plot for the optimization of $12 \mathrm{~L} 14$ free machining steel turning

Fig. 2 compares both optimal solutions with the feasible region for this problem. As easily noticed, the optimal point obtained with the conventional GCM, although seems a good solution, was established out of the feasible region. On the other hand, the solution found by GCM based on principal components, in addition to showing better practical results, was able to locate the optimal point inside the feasible region. The main argument for this fact is because the correlation structure of responses was considered in the second analysis. Thus, when correlations exist and are significant, the use of optimization strategies that do not consider this information can conduct the problem to solutions that do not represent the best process condition.

\section{CONCLUSIONS}

This work presented the Global Criterion Method based on principal components as an alternative to optimize manufacturing processes with multiple correlated responses. From previous analysis, it was observed that the correlations are important information for this kind of problem and its negligence can direct the optimal point to inappropriate locations.

The GCM based on principal components was successfully applied to the optimization of $12 \mathrm{~L} 14$ free machining steel turning process. An optimized condition with good surface finishing and good material removal was found for a cutting speed of 212 $\mathrm{m} / \mathrm{min}$, feed rate of $0.13 \mathrm{~mm} / \mathrm{rev}$ and depth of cut of $1.33 \mathrm{~mm}$. All optimized responses were established within the defined specification limits.

In comparison to the optimal point obtained with the conventional technique, the GCM based on principal components showed an optimal solution with better practical results in terms of roughness and material removal, but with a higher cutting time. In relation to the feasible region of the problem, the GCM based on principal components directed the optimal point to inside this region, while the solution found by conventional GCM stayed out of it. Due to these reasons, the optimal point found with GCM based on principal components was characterized as a more adequate solution.

Although the technique presented in this work has been effective to the optimization of $12 \mathrm{~L} 14$ free machining steel turning, it needs to be tested in other processes. Therefore, it is suggested for future research works that this strategy is applied and verified in others turning applications and other manufacturing operations, like milling, cutting or welding.

\section{ACKNOWLEDGEMENTS}

The authors acknowledge the Capes, CNPq, FAPEMIG and the Institute of Mechanical Engineering of UNIFEI for supporting this work.

\section{REFERENCES}

[1] Chen, L.H. (1997). Designing robust products with multiple quality characteristics. Computers \& Operations Research, vol. 24, no. 10, p. 937-944, DOI:10.1016/S0305-0548(97)00003-8. 
[2] Župerl, U., Čuš, F. (2008). Machining process optimization by colony based cooperative search technique. Strojniški vestnik - Journal of Mechanical Engineering, vol. 54, no. 11, p. 751-758.

[3] Derringer, G., Suich, R. (1980). Simultaneous optimization of several response variables. Journal of Quality Technology, vol. 12, no. 4, p. 214-219.

[4] Chiao, H., Hamada, M. (2001). Analyzing experiments with correlated multiple responses. Journal of Quality Technology, vol. 33, no. 4. p. 451-465.

[5] Ch'ng, C.K., Quah, S.H., Low, H.C. (2005). Index $\mathrm{Cpm}$ in multiple response optimization. Quality Engineering, vol. 17, no. 1, p. 165-171, DOI:10.1081/ QEN-200029001.

[6] Plante, R.D. (2001). Process capability: a criterion for optimizing multiple response product and process design. IIE Transactions, vol. 33, no. 5, p. 497-509, DOI:10.1080/07408170108936849.

[7] Rao, S.S. (1996). Engineering optimization: theory and practice. $3^{\text {rd }}$ ed. John Wiley \& Sons, New Jersey.

[8] Khuri, A.I., Conlon, M. (1981). Simultaneous optimization of multiple responses represented by polynomial regression functions. Technometrics, vol. 23, no. 4, p. 363-375, DOI:10.2307/1268226.

[9] Bratchell, N. (1989). Multivariate response surface modeling by Principal Components Analysis. Journal of Chemometrics, vol. 3, no.4, p. 579-588, DOI:10.1002/cem.1180030406.

[10] Paiva, A.P., Paiva, E.J., Ferreira, J.R., Balestrassi, P.P., Costa, S.C. (2009). A multivariate mean square error optimization of AISI 52100 hardened steel turning. International Journal of Advanced Manufacturing Technology, vol. 43, no. 7-8, p. 631643, DOI:10.1007/s00170-008-1745-5.
[11] Wang, F.K., Du, T.C.T. (2000). Using Principal Component Analysis in process performance for multivariate data. Omega, vol. 28, no. 2, p. 185-194, DOI:10.1016/S0305-0483(99)00036-5.

[12]Rossi, F. (2001). Blending response surface methodology and principal components analysis to match a target product. Food Quality and Preference, vol. 12, no. 5, p. 457-465, DOI:10.1016/ S0950-3293(01)00037-4.

[13] Montgomery, D.C. (2005). Design and Analysis of Experiments. $6^{\text {th }}$ ed.: John Wiley, New York..

[14] Ficko, M., Brezocnik, M., Balic, J. (2005). A model for forming a flexible manufacturing system using genetic algorithms. Strojniški vestnik - Journal of Mechanical Engineering, vol. 51, no. 1, p. 28-40.

[15]Busacca, G.P., Marseguerra, M., Zio, E. (2001). Multiobjective optimization by Genetic Algorithms: Application to safety systems. Reliability Engineering \& System Safety, vol. 72, no. 1, p. 5974, DOI:10.1016/S0951-8320(00)00109-5.

[16] Johnson, R.A., Wichern, D. (2002). Applied Multivariate Statistical Analysis. 5th ed. PrenticeHall, New Jersey.

[17] Ronggen, Y., Ren, M. (2011). Wavelet denoising using principal component analysis. Expert Systems with Applications, vol. 38, no. 1, p. 1073-1076, DOI:10.1016/j.eswa.2010.07.069.

[18]Ho, C.T.B., Wu, D.D. (2009). Online banking performance evaluation using data envelopment analysis and Principal Component Analysis. Computers \& Operations Research, vol. 36, no. 6, p. 1835-1842, DOI:10.1016/j.cor.2008.05.008. 